\title{
Advective collisions
}

\author{
B. Andersson ${ }^{1}$, K. Gustavsson ${ }^{1}$, B. Mehlig ${ }^{1}$, and M. Wilkinson ${ }^{2}$ \\ ${ }^{1}$ Department of Physics, Göteborg University, \\ 41296 Gothenburg, Sweden \\ ${ }^{2}$ Faculty of Mathematics and Computing, \\ The Open University, Walton Hall, \\ Milton Keynes, MK7 6AA, England
}

\begin{abstract}
Small particles advected in a fluid can collide (and therefore aggregate) due to the stretching or shearing of fluid elements. This effect is usually discussed in terms of a theory due to Saffman and Turner (J. Fluid Mech., 1, 16-30, (1956)). We show that in complex or random flows the Saffman-Turner theory for the collision rate describes only an initial transient (which we evaluate exactly). We obtain precise expressions for the steady-state collision rate for flows with small Kubo number, including the influence of fractal clustering on the collision rate for compressible flows. For incompressible turbulent flows, where the Kubo number is of order unity, the Saffman-Turner theory is an upper bound.

PACS numbers: 45.50.Tn, 92.60.Mt, 05.40.-a, 05.45.-a, 02.50.-r
\end{abstract}

1. Introduction. Suspensions of small particles in a fluid, such as aerosols or colloids, are ubiquitous in the natural world and in technology. Such systems may be unstable due to collisions of the suspended particles giving rise to aggregation or to chemical reaction. These collision processes are fundamental to understanding the formation of raindrops from clouds [1], or the growth of planets from dust in a protostellar environment [2].

Collisions between particles will always occur due to Brownian diffusion. The rate of collision may be greatly increased if the fluid is in macroscopic motion [3], such as turbulence [4]. If the inertia of the suspended particles is significant, the suspended particles may move relative to the fluid and this effect can greatly increase the rate of collisions [5, 6, 7]. However if the particles are sufficiently small that the effect of their inertia is negligible (advective limit), the particles may be brought into contact by the effect of shearing motion in the flow. This process was discussed by Saffmann and Turner [4] and their theory has been the starting point for most subsequent work on advective collisions in smooth flows. Here we point out a previously unremarked assumption in [4], which implies that their expression for the collision rate describes a short-lived transient. In the case of incompressible flow their expression is an upper bound: we give the first precise results on the steady-state collision rate of their model. Both the Saffman-Turner theory and our expression are compared with numerical simulations in Fig. 1 $\mathbf{a}, \mathbf{b}$ (for synthetic incompressible flows in two and three dimensions respectively; the details are explained later). In both cases we observe an initial transient given by the Saffman-Turner theory, and at larger times the collision rate approaches a significantly lower value which is in agreement with our theory.

Reference [4] treats the local flow in the vicinity of a particle as if it were a steady hyperbolic flow, whereas in reality it must fluctuate. We argue that the appropriate theory for the collision rate must depend upon a dimensionless parameter termed the Kubo number, Ku.

2. Summary. In this letter we give the first precise expressions for advective collisions in smooth flows. We obtain asymptotic results in the case of flows with small Kubo number, in both two and three dimensions, including flows with Richardson diffusion and compressible flows where particles cluster onto a fractal set. We also describe new results on the evaluation of the SaffmanTurner expression for the initial collision rate in two and three dimensions.

3. A formula for the collision rate. We start by discussing a general formula for the collision rate, which is very similar to that proposed by Saffmann and Turner [4]. We model processes where particles coalesce upon contact with probability unity. The particles collide when their separation falls below $2 a$ (where $a$ is the particle radius). For simplicity we initially discuss two spatial dimensions. Let $v_{r}(r, \theta, t)$ be the radial component of the velocity of the fluid relative to a test particle at $(r, \theta)$ (in polar coordinates). We make the approximation that the velocity field is not perturbed by the suspended particles: this is valid in the Epstein low-pressure regime [8] and is a reasonable approximation [4] in conventional fluids. The collision rate for a single particle is then the rate at which points distributed randomly with density $n_{0}$ cross into the disc of radius $2 a$ centered on the origin. The instantaneous collision rate may then be written as

$$
R=-2 a n_{0} \int_{0}^{2 \pi} \mathrm{d} \theta v_{r}(2 a, \theta, t) \Theta\left(-v_{r}(2 a, \theta, t)\right) \chi(2 a, \theta, t)
$$

where the Heaviside function $\Theta(\cdot)$ selects for particles entering a disc of radius $2 a, n_{0}$ is the number density of the suspended particles in the vicinity of the test particle, and $\chi(r, \theta, t)$ is zero if the fluid at $(r, \theta)$ at time $t$ 

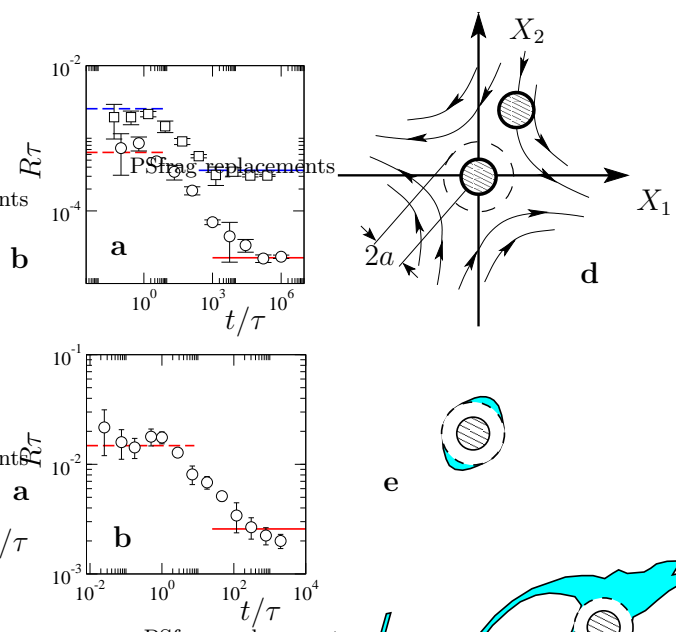

e
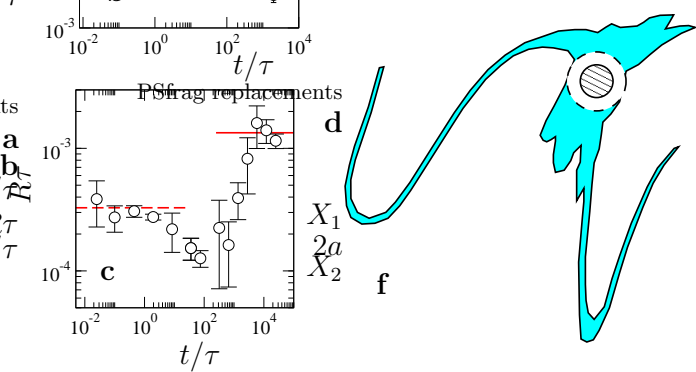

FIG. 1: Collision rate as a function of time for particles advected in an random flow. a incompressible flow in two spatial dimensions, $u_{0}=1, \eta=0.1, n_{0}=10^{3}, \tau=0.001$ (o) and $\tau=0.004(\square), a=0.003$. Theory for initial transient (11), dashed lines, for collision rate (9), full lines. Error bars correspond to one standard deviation. $\mathbf{b}$ three spatial dimensions, $u_{0}=1, \tau=0.004, \eta=0.1, a=0.02$, and $n_{0}=2 \times 10^{3}$. Theory for initial transient (18), for collision rate (17). $\mathbf{c}$ twodimensional compressible flow. Parameters: $u_{0}=1, \eta=0.1$, $\tau=0.004, a=0.001, n_{0}=10^{3}$, and $\Gamma=2$. Initial transient (16), dashed line; collision rate (15), solid line. d steady hyperbolic flow in the vicinity of a test particle, e shows region (in rest frame of test particle) occupied by particles which will collide with test particle after time $t=20 \tau$ (parameters as in Fig. 1a, $\square$ ), f same but after time $t=10^{3} \tau$.

has previously passed through the disc of radius $2 a$, and unity otherwise. The collision rate of a single particle $R_{\mathrm{a}}$ is the time-average of (11), yielding an exact and general expression for the advective collision rate, but in the general case it is difficult to evaluate since the characteristic function $\chi$ depends on the history of the flow.

The relative speed $v_{r}$ is determined as follows [4]: for small particles $a \ll \eta$ we approximate the flow $u(\boldsymbol{r}, 0)$ in the vicinity of the test particle by a linearised equation for the small distance $\boldsymbol{X}$ from the test particle: $\dot{\boldsymbol{X}}=$ $\mathbf{A}(\mathbf{0}, 0) \boldsymbol{X}$, where $\mathbf{A}(\boldsymbol{r}, t)$ is the strain matrix of the flow $\boldsymbol{u}(\boldsymbol{r}, t)$. We then have $v_{r}=\dot{\boldsymbol{X}} \cdot \hat{\mathbf{n}}(\theta)$ where $\hat{\mathbf{n}}(\theta)$ is a unit vector normal to the surface at a point with polar angle $\theta$. At the point $\boldsymbol{X}=2 a \hat{\mathbf{n}}(\theta)$ on the surface of the disc

$$
v_{r}=2 a \hat{\mathbf{n}} \cdot \mathbf{A} \hat{\mathbf{n}} .
$$

Eqs (12) appear as the key expressions for the collision rate in [4], but without the factor $\chi$. Ref. [4] describes the flow in the vicinity of a test particle as if it were a steady hyperbolic flow (Fig. 1 d). In that case, particles advected by the local flow only pass through the circle $r=2 a$ once (Fig. 1) , so that the factor $\chi$ has no effect. In general, however, the flow in the vicinity of the test particle will fluctuate, as illustrated in Fig. 1f, so that the directions of the eigenvectors of $\mathbf{A}$ rotate. The function $\chi(r, \theta, t)$ equals zero for regions where the flow at $r=2 a$ has reversed direction, so that points that were originally advected away return to the surface. This reduces the collision rate below the Saffman-Turner estimate.

There is one limit in which the Saffman-Turner theory is exact: this is the limit of very short times, when the initial condition ensures that $\chi(r, \theta, 0)=1$. For later times, it might be expected that the Saffman-Turner theory is an upper bound on the collision rate. This is true if the flow field is incompressible. For compressible flows there is a complication, because particles may cluster together, and the density in the vicinity of a test particle may be larger than the average density, $n_{0}$.

Although it is not possible to make a precise theory for the steady-state collision rate in the general case, this can be done in the limit where the velocity field of the fluid is rapidly fluctuating. The effect of fluctuations depends on a dimensionless parameter termed the Kubo number. A random or turbulent flow may be characterised by a correlation length $\eta$, a correlation time $\tau$, and a characteristic velocity $u_{0}$. The Kubo number $\mathrm{Ku}=u_{0} \tau / \eta$ is a dimensionless measure of how rapidly the flow fluctuates. It might be argued that the Saffman-Turner theory, which treats the flow as if it were steady, would apply to flows with very large Kubo number. However, such flows are unphysical, because the velocity field transports spatial fluctuations so that they become temporal fluctuations, implying that the Kubo number is never large. In the case of fully-developed turbulence, $\mathrm{Ku}=O(1)$.

4. Collision rate for small Kubo number. In the limit $\mathrm{Ku} \rightarrow 0$ (that is, where the correlation time approaches zero), the relative separation of two particles undergoes a diffusion process determining the collision rate.

We consider the case of two spatial dimensions. By homogeneity we can assume that the particles are at $\mathbf{0}$ and $\boldsymbol{X}=\left(X_{1}, X_{2}\right)$ and by isotropy we can take $X_{2}=0$ and write $\boldsymbol{X}=(r, 0)$. The fluctuation $\delta X_{i}$ of the separation in a short time $\delta t$ satisfies $\left\langle\delta X_{i}\right\rangle=0$. The correlation of these increments is $\left\langle\delta X_{i} \delta X_{j}\right\rangle=2 D_{i j} \delta t$, with

$$
D_{i j}=\frac{1}{2} \int_{-\infty}^{\infty} \mathrm{d} t\left\langle\left[u_{i}(\boldsymbol{X}, \mathrm{t})-u_{i}(\mathbf{0}, t)\right]\left[u_{j}(\boldsymbol{X}, 0)-u_{j}(\mathbf{0}, 0)\right]\right\rangle .
$$

These 'diffusion constants' are computed as follows. We consider a velocity field $\boldsymbol{u}(\boldsymbol{r}, t)$ generated by a 'stream function' $\psi(\boldsymbol{r}, t): \boldsymbol{u}=\boldsymbol{\nabla} \wedge \psi \hat{\mathbf{n}}_{z}$, where $\hat{\mathbf{n}}_{z}$ is a unit vector in the $z$-direction, with correlation function $\langle\psi(\boldsymbol{r}, t) \psi(\mathbf{0}, 0)\rangle=C(r, t)$, where $r=|\boldsymbol{r}|$. For $\boldsymbol{X}=(r, 0)$ 
we find $D_{12}=D_{21}=0$ and

$$
\begin{aligned}
& D_{11}(r)=-\int_{-\infty}^{\infty} \mathrm{d} t\left[C^{\prime \prime}(0, t)-\frac{1}{r} C^{\prime}(r, t)\right] \\
& D_{22}(r)=-\int_{-\infty}^{\infty} \mathrm{d} t\left[C^{\prime \prime}(0, t)-C^{\prime \prime}(r, t)\right]
\end{aligned}
$$

where the prime denotes a derivative w.r.t. $r$.

We set up a Fokker-Planck equation for the separation $r$ of two particles. The change in the value of $r$ in $\delta t$ is $\delta r=\sqrt{(x+\delta x)^{2}+\delta y^{2}}-x=\delta x+\delta y^{2} /(2 x)$, so that $\langle\delta r\rangle=D_{22}(r) \delta t / r$ and $\left\langle\delta r^{2}\right\rangle=\left\langle\delta x^{2}\right\rangle=2 D_{11}(r) \delta t$. We obtain the Fokker-Planck equation

$$
\frac{\partial \rho}{\partial t}=\frac{\partial}{\partial r}\left[-\frac{D_{22}(r) \rho}{r}+\frac{\partial}{\partial r}\left(D_{11}(r) \rho\right)\right] .
$$

The collision rate is determined by a steady-state solution of (5) with a constant flux $-J$. The boundary condition is $\rho(2 a)=0$, which corresponds to particles which reach $r=2 a$ being absorbed, so that there are no re-collisions. The collision rate is $R_{\mathrm{a}}=J$. To enforce the boundary condition at $r=2 a$, it is sufficient to consider an approximate solution valid at small values of $r$. Expanding the diffusion constants for small $r$ we find $D_{11}(r) \sim \mathcal{D} r^{2}$ and $D_{22}(r) \sim 3 \mathcal{D} r^{2}$, with

$$
\mathcal{D}=\frac{1}{6} \int_{-\infty}^{\infty} \mathrm{d} t C^{\prime \prime \prime \prime}(0, t)
$$

we obtain the desired steady-state solution

$$
\rho(r)=J /(2 \mathcal{D} r)+A r .
$$

To determine the constant $A$ we match the solution (7) for $J=0$ to the exact steady-state zero-flux solution of (5). The latter satisfies

$$
\frac{\partial \log \rho}{\partial r}-\frac{1}{r}=\frac{1}{D_{11}}\left[\frac{D_{22}}{r}-\frac{\partial D_{11}}{\partial r}\right]-\frac{1}{r} \equiv \alpha(r) .
$$

From (41) we find $\alpha(r)=0$. The density is thus proportional to $r$, normalisation gives $\rho(r)=2 \pi n_{0} r$. We conclude that $A=2 \pi n_{0}$ in (7). The collision rate is determined by setting $\rho(2 a)=0$ in (77) and solving for $R_{\mathrm{a}}=J$ :

$$
R_{a}=16 \pi \mathcal{D} n_{0} a^{2} .
$$

5. Richardson diffusion. In a steady, fully developed turbulent flow [9], particle separations exhibit Richardson diffusion $\mathrm{d}\left\langle r^{2}\right\rangle / \mathrm{d} t \propto r^{4 / 3}$ in the inertial range $r \gg \eta$ [10] (where $\eta$ is the Kolmogorov length). This implies $D_{i j}(r) \propto r^{4 / 3}$. It is of interest to consider the collision rate in a flow exhibiting Richardson diffusion in the limit $\mathrm{Ku} \rightarrow 0$, to establish whether additional complications arise when considering a multiscale flow (although real turbulence has $\mathrm{Ku}=O(1)$, and is at the borderline of applicability of our theory). In the following we argue that (9) is unchanged for a flow exhibiting Richardson diffusion, such that $D_{i j}(r) \sim r^{\xi}$ in the inertial range.

At first sight, given eq. (5), this power-law scaling of the diffusion constants suggests that there should be a power-law behaviour of $\rho(r)$ in the inertial range, with an exponent which depends upon $\xi$. This would be inconsistent with the requirement that $\rho(r) \sim 2 \pi n_{0} r$ for $r \gg \eta$ (as it must be in a homogenous and isotropic flow). However, given equations (4), we see that $C(r, t)$ contains a term which can be approximated by a multiple of $r^{2+\xi}$ for $r$ in the inertial range. It follows that the coefficients of $D_{11}$ and $D_{22}$ are related, $D_{11}(r)=C r^{\xi}$ and $D_{22}(r)=C(1+\xi) r^{\xi}$. Comparison with (5) shows that $\rho \sim r$ in the inertial range, as expected. Since the velocity field in two dimensions is generated by a stream function, it follows immediately that $\alpha(r)=0$, and thus $\rho(r)=2 \pi n_{0} r$ for $r \ll \eta$ as well as for $r \gg \eta$. We conclude that our result (9) for the collision rate of particles advected in incompressible flows at small values of $\mathrm{Ku}$ is insensitive to what happens in the inertial range.

6. Computer simulations. The numerical simulations shown in Figs. 1 $\mathbf{a}$ and $\mathbf{b}$ employ a Gaussian random stream function with spatially and temporally stationary, spatially isotropic statistics with mean value zero and $C(r, t)=\left(u_{0}^{2} \eta^{2} / 2\right) \exp \left[-|t| / \tau-r^{2} /\left(2 \eta^{2}\right)\right]$, for which $\mathcal{D}=u_{0}^{2} \tau /\left(2 \eta^{2}\right)$. The particles are initially randomly positioned, and they are regarded as having collided when their separation falls below $2 a$. Our plots show the rate of collision with a test particle which is also advected by the velocity field. Upon collision with the test particle, colliding particles are removed. The collision rate was determined by dividing by $t$ the number of particles which collided in the time interval $[0, t]$. This yields accurate results for the initial transient, $R_{0}$, and for the steady-state collision rate, $R_{\mathrm{a}}$. Only a small fraction of particles had collided by the end of each run (less than a few percent). Fig. 1 1 a shows the collision rate as a function of time for particles advected in a two-dimensional incompressible flow for small Kubo number. We observe good agreement with the analytical result (9), full line. In the following we show how to derive the initial transients shown in Fig. 1 a, dashed lines.

7. Exact result for the initial transient. The initial transient is obtained by averaging equations (11), (2) over the ensemble of random strain matrices determined by the statistics of the stream function $\psi(\mathbf{0}, 0)$. In order to compute the average of $R$, we decompose the matrix A which appears in (2) into a symmetric $\mathbf{A}_{()}$and an antisymmetric $\mathbf{A}_{[]}$part and sort the eigenvalues $\sigma_{i}, i=$ $1, \ldots, d$ of the symmetric part in order: $\sigma_{1} \leq \sigma_{2} \leq, \ldots, \leq$ $\sigma_{d}$. In two dimensions we obtain for a given $\mathbf{A}$

$$
R\left(\sigma_{2}\right)=8 n_{0} a^{2} \sigma_{2} .
$$

We average over the distribution of the largest eigenvalue of $\mathbf{A}_{()}, P\left(\sigma_{2}\right)=\left(2 \sigma_{2} \eta^{2} / u_{0}^{2}\right) \exp \left[-\sigma_{2}^{2} \eta^{2} / u_{0}^{2}\right]$, where $\sigma_{2} \geq$ 
0 , and find for the initial rate of collision, $R_{0}$ :

$$
R_{0}=4 \sqrt{\pi} n_{0} a^{2} u_{0} / \eta
$$

This initial transient is shown in Fig. 1 a (dashed lines). Comparing with (9), we have $R_{\mathrm{a}} \sim \mathrm{Ku} R_{0}$, demonstrating that the effect of eliminating multiple collisions is significant when the velocity field $\boldsymbol{u}(\boldsymbol{r}, t)$ is rapidly fluctuating.

8. Collision rate in the presence of fractal clustering. When the velocity field has a compressible component, the particles cluster onto a fractal set [11]. This may alter the collision rate, because particles which cluster together are expected to collide more frequently [6]. Here we present the first exact results on this effect. When considering compressibility effects we restrict ourselves to two spatial dimensions, because the natural experimental realisation of particles advected by compressible flows involves particles floating on the surface of a turbulent fluid, such as the experiments discussed in [11, 12].

We consider a partially compressible flow of the form $\boldsymbol{u}=\left(\boldsymbol{\nabla} \wedge \psi \hat{\mathbf{n}}_{z}+\beta \boldsymbol{\nabla} \phi\right) / \sqrt{1+\beta^{2}}$ where $\psi$ and $\phi$ are independent Gaussian random functions with correlation function $C(r, t)$ and $\beta>0$. As before we seek a solution of (5) with constant flux $-J$. We have

$$
\begin{aligned}
& D_{11}(r)=-\int_{-\infty}^{\infty} \mathrm{d} t\left[C^{\prime \prime}(0, t)-\frac{C^{\prime}(r, t)}{r\left(1+\beta^{2}\right)}-\frac{\beta^{2} C^{\prime \prime}(r, t)}{1+\beta^{2}}\right] \\
& D_{22}(r)=-\int_{-\infty}^{\infty} \mathrm{d} t\left[C^{\prime \prime}(0, t)-\frac{C^{\prime \prime}(r, t)}{1+\beta^{2}}-\frac{\beta^{2} C^{\prime}(r, t)}{r\left(1+\beta^{2}\right)}\right]
\end{aligned}
$$

so that $D_{22} / D_{11} \rightarrow \Gamma \equiv\left(\beta^{2}+3\right) /\left(3 \beta^{2}+1\right)$ as $r \rightarrow 0$. The steady-state solution of (5) is for $r \ll \eta$

$$
\rho(r)=[(\Gamma+1) /(\Gamma-1)] J /(4 \mathcal{D} r)+A r^{\Gamma-2} .
$$

The constant $A$ is determined, as before, by matching to the small- $r$ behaviour of the exact zero-flux solution of (8) with the diffusion constants (12):

$$
\rho(r)=2 \pi n_{0} r \exp \left[-\int_{r}^{\infty} \mathrm{d} r^{\prime} \alpha\left(r^{\prime}\right)\right] .
$$

In order to match (13) with (14) we evaluate (14) for small values of $r$. One obtains $\rho(r) \sim 2 \pi n_{0} \eta(r / \eta)^{D_{2}-1}$ where $D_{2}=\Gamma-1$ is the correlation dimension of the fractal set onto which the particles cluster (this dimension was first determined in Ref. [13]). Matching with (13) we obtain (for $1<\Gamma \leq 3$ )

$$
R_{\mathrm{a}}=[(\Gamma-1) /(\Gamma+1)] 8 \pi \mathcal{D} n_{0} \eta^{2}(2 a / \eta)^{D_{2}} .
$$

For the initial transient in compressible flows we find:

$$
R_{0}=(8 \sqrt{\pi} / \sqrt{1+\Gamma}) n_{0} a^{2} u_{0} / \eta .
$$

Comparison with (15) shows that eq. (1) no longer gives an upper bound of the collision rate upon setting $\chi=$ 1. This is because Eqs. (11) and (11) assume a uniform distribution of particles. But for times $t \gg\left|\lambda_{1}+\lambda_{2}\right|^{-1}$ (here $\lambda_{1}>\lambda_{2}$ are the Lyapunov exponents of the flow), the particles cluster on a fractal set 11] which can lead to an increase in the collision rate (Fig. 1c).

There is a critical point at $\Gamma=1$ where $\lambda_{1}$ becomes negative and path-coalescence occurs [14]. Experimental evidence indicates that $\Gamma=1$ for surface flows above a turbulent fluid [12]. At this critical point we obtain $R_{\mathrm{a}}=4 \pi \mathcal{D} n_{0} \eta^{2} \log (2 a / \eta)$.

9. Three spatial dimensions. We consider a threedimensional incompressible flow generated by three random vector fields [15]. The calculation of $R_{\mathrm{a}}$ proceeds in a fashion similar to the derivation of (9):

$$
R_{\mathrm{a}}=96 \pi \mathcal{D} n_{0} a^{3}, \quad \mathcal{D}=\left.\frac{1}{2} \frac{\mathrm{d}^{2} D_{11}(r)}{\mathrm{d} r^{2}}\right|_{r=0} .
$$

As in the two-dimensional case we find that Richardson diffusion does not alter the result (17). The initial transient in three dimensions is derived as outlined above. We have obtained an explicit expression for $R\left(\sigma_{2}, \sigma_{3}\right)$ in terms of the largest eigenvalues $\sigma_{2}, \sigma_{3}$ of $\mathbf{A}_{()}$, but could not perform the average of $\sigma_{2}, \sigma_{3}$ analytically. Numerical averaging for our Gaussian-correlated model gives

$$
R_{0} \approx 24 n_{0} a^{3} u_{0} / \eta
$$

Results (17) and (18) are shown in Fig. 1b in comparison with numerical simulations.

Acknowledgements. We acknowledge support from Vetenskapsrådet and from 'Nanoparticles in an interactive environment' at Göteborg university.

[1] R. A. Shaw, Annu. Rev. Fluid Mech., 35, 183, (2003).

[2] P. J. Armitage, astro-ph/0701485.

[3] M. v. Smoluchowsky, Zeitschrift f. physik. Chemie, XCII 129-168, (1917); see eq. (28) on p. 156.

[4] P. G. Saffman and J. S. Turner, J. Fluid Mech., 1, 16-30, (1956).

[5] J. Abrahamson, Chem. Eng. Sci., 30, 1371-9, (1975).

[6] G. Falkovich, A. Fouxon and G. Stepanov, Nature, 419, 151-154, (2002).

[7] M. Wilkinson, B. Mehlig and V. Bezuglyy, Phys. Rev. Lett., 97, 048501, (2006).

[8] P. S. Epstein, Phys. Rev., 23, 710, (1924).

[9] U. Frisch, Turbulence, Cambridge University Press, (1997).

[10] L. F. Richardson, Proc. Roy. Soc. Lond., A110, 709-37, (1926).

[11] J. Sommerer and E. Ott, Science, 359, 334, (1993).

[12] J. R. Cressman, J. Davoudi, W. I. Goldberg and J. Schumacher, New J. Phys., 6, 53, (2004).

[13] E. Balkovsky, G. Falkovich, and A. Fouxon, Phys. Rev. Lett., 86, 2790, (2001); cond-mat/9912027.

[14] B. Mehlig and M. Wilkinson, Phys. Rev. Lett, 92, 250602, (2004).

[15] K. P. Duncan, B. Mehlig, S. Östlund and M. Wilkinson, Phys. Rev. Lett., 95, 240602, (2005). 